\title{
AVALIAÇÃO MICROBIOLÓGICA DO QUEIJO TIPO MUSSARELA E QUEIJO COLONIAL COMERCIALIZADO NA REGIÃO OESTE DO PARANÁ
}

\section{MICROBIOLOGICAL EVALUATION OF MOZZARELLA CHEESE KIND AND COLONIAL CHEESE MARKETED IN THE WEST REGION OF PARANÁ}

\author{
Francielly T. Dos Santos-Koelln ${ }^{1}$; Ademir Mattana²; Eliane Hermes ${ }^{3}$ \\ ${ }^{1,2}$ Universidade Tecnológica Federal do Paraná - UTFPR - Medianeira - Brasil \\ franciellykoelln@yahoo.com.br \\ ${ }^{3}$ Universidade Estadual do Oeste do Paraná - UNIOESTE - Cascavel - Brasil \\ elianehermes@yahoo.com.br
}

\begin{abstract}
Resumo
Tendo em vista o grande consumo dos queijos tipo Mussarela e queijo Colonial faz-se necessário à avaliação da qualidade microbiológica destes. O estudo objetivou-se em avaliar a qualidade microbiológica de sete amostras de queijo tipo Mussarela e sete amostras de queijo Colonial comercializados na região oeste do Paraná. Sendo coletadas em dois períodos distintos totalizando 28 amostras, realizando-se as seguintes análises microbiológicas: Contagem de Coliformes a 45 ${ }^{\circ} \mathrm{C}$, Contagem de Staphylococcus coagulase positiva e Salmonella sp. onde as amostras analisadas do queijo tipo Mussarela encontravam-se dentro dos limites estabelecidos pela legislação vigente, para o queijo Colonial as amostras encontram-se fora do limite.
\end{abstract}

Palavras-chave: coliformes, legislação vigente, queijo.

\section{Introdução}

O queijo é um dos principais alimentos desde 7000 a.C. produzido na região localizada entre os rios Tigres e Eufrates. A tendência atual da atividade da indústria é de elaborar novos métodos para o tratamento da matéria-prima nos quais se possam obter os maiores benefícios. O produtor de queijos deve procurar que este seja comestível, aceitável, comerciável e que constitua um alimento nutritivo e livre de contaminação por microrganismos indesejáveis (DREYFUSSO et al., 2001).

O leite destinado à fabricação de queijos deve apresentar características que objetivam a uniformização do produto final. A porcentagem de gordura é variável para cada tipo de queijo, por isso, torna-se necessária a padronização da mesma no leite visando atender os padrões tecnológicos dos queijos (CARVALHO \& ALBUQUERQUE, 1996). 
O queijo tipo Mussarela é um queijo de massa filada, de origem italiana, porém conhecido, produzido, apreciado e consumido no mundo todo, e especialmente no Brasil, onde se destaca como sendo o segundo queijo mais fabricado, representando cerca de 20 \% da produção total de queijos. A maior utilização e consumo do queijo Mussarela são como ingrediente para a confecção de inúmeros pratos quentes, sanduíches, pizzas, entre outros alimentos, que visam explorar as suas propriedades para fatiamento e a sua facilidade de derretimento (VALLE, 2004).

O queijo Colonial tem importância na vida econômica da população de colonos que residem no meio rural, que tem nessa atividade uma fonte alternativa de renda. Não existe padrão de técnicas de fabricação entre as fazendas produtoras, e a elevada contaminação por coliformes ocorre porque a qualidade higiênico-sanitária é muito precária, constituindo um risco em potencial para a saúde do consumidor. Esses queijos, produzidos no meio rural, são feitos com leite cru e, muitas vezes, sem cuidado higiênico na sua elaboração, mas em função da sua composição química, é considerado um alimento importante nutricionalmente (LUCAS, 2008).

Segundo FURTADO (1999), quando a contaminação é grande os coliformes são facilmente notados pelo número de pequenos olhos distribuídos na massa fermentada, logo quando a massa é filada, uma grande parte desta flora gasógena é eliminada e as olhaduras desaparecem na massa moldada a quente, mas isto não quer dizer que a presença maciça de coliformes foi reduzida, sendo que o sabor da Mussarela é alterado, pois a fermentação por coliformes produz compostos diversos, como ácido acético, que ficam no queijo.

O Staphyfococcus aureus e a Salmonella sp. fazem parte do grupo de microrganismos agentes de toxinfecções alimentares, as Salmonellas $s p$. que causam intoxicações alimentares são classificadas em mais de 2000 sorotipos capazes de invadir e infectar o corpo do homem e dos animais. A pele das mãos e o nariz freqüentemente abrigam estafilococos, alguns dos quais produzem toxinas nos alimentos cozidos indicando condições de fabricação inadequadas. Surtos de intoxicações alimentares estafilococicas ocorrem em hospitais e fábricas através de queijos classificados como de segunda classe devido às falhas na cultura iniciadora resultando em flavour anormal. A pasteurização do leite para a produção de queijos reduz, senão elimina os riscos de toxinfecções alimentares (HOBBS, 1998).

Tendo em vista todos os agravantes para a saúde pela contaminação por estes microrganismos, o presente trabalho teve como objetivo avaliar microbiologicamente queijos do tipo Mussarela e queijo Colonial comercializados na região Oeste do Paraná. 


\section{Material e Métodos}

Foram analisadas no período de agosto de 2008 a fevereiro de 2009, sete amostras de queijo tipo mussarela sendo denominadas de A, B, C, D, E, F e G e sete amostras de queijo Colonial denominadas de $\mathrm{H}, \mathrm{I}, \mathrm{J}, \mathrm{K}, \mathrm{L}, \mathrm{M}$ e $\mathrm{N}$. As amostras foram adquiridas em supermercados da região oeste do Paraná. Para a realização das análises as amostras de queijo foram transportadas em recipientes isotérmicos até o Laboratório de Análises Microbiológicas e Físico-químicas de Alimento e Água - LAMAG, da Universidade Tecnológica Federal do Paraná (UTFPR), Campus de Medianeira.

As análises microbiológicas realizadas nos dois tipos de queijo foram: Contagem de Coliformes a $45{ }^{\circ} \mathrm{C}$, Contagem de Staphylococcus coagulase positiva e Salmonella sp, sendo realizadas segundo a Instrução Normativa ${ }^{\circ}$ 62, de 26 de agosto de 2003 (BRASIL, 2003). Para cada marca analisada realizaram-se duas repetições sendo 14 análises do queijo tipo Mussarela e 14 análises do queijo Colonial totalizando 28 análises.

\section{Resultados e Discussão}

Na Tabela 1 estão expressos os resultados das análises de contagem de Coliformes a $45^{\circ} \mathrm{C}$ e Contagem de Staphylococcus coagulase positiva do queijo tipo Mussarela das sete marcas avaliadas.

Tabela 1 - Análise Microbiológica de Coliformes a $45^{\circ} \mathrm{C}$ e Staphylococcus coagulase positiva, de queijos tipo Mussarela comercializados na região oeste do Paraná

\begin{tabular}{|c|c|c|c|c|}
\hline \multirow[t]{2}{*}{ Amostra } & \multicolumn{2}{|c|}{$\begin{array}{c}\text { Análise de Coliformes } \\
\text { a } 45^{\circ} \mathrm{C} \\
\end{array}$} & \multicolumn{2}{|c|}{$\begin{array}{c}\text { Análise de Staphylococcus } \\
\text { coagulase positiva }\end{array}$} \\
\hline & $1^{\circ}$ repetição & $2^{\circ}$ repetição & $1^{\circ}$ repetição & $2^{\circ}$ repetição \\
\hline A & $<10^{2} \mathrm{UFC} / \mathrm{g}$ & $<10^{2} \mathrm{UFC} / \mathrm{g}$ & $<10^{2} \mathrm{UFC} / \mathrm{g}$ & $<10^{2} \mathrm{UFC} / \mathrm{g}$ \\
\hline B & $<10$ UFC/g & $<10$ UFC/g & $<10$ UFC/g & $<10 \mathrm{UFC} / \mathrm{g}$ \\
\hline $\mathrm{C}$ & $<10^{2} \mathrm{UFC} / \mathrm{g}$ & $<10^{2} \mathrm{UFC} / \mathrm{g}$ & $<10^{2} \mathrm{UFC} / \mathrm{g}$ & $<10^{2} \mathrm{UFC} / \mathrm{g}$ \\
\hline $\mathrm{D}$ & $<10^{2} \mathrm{UFC} / \mathrm{g}$ & $<10$ UFC/g & $<10^{2} \mathrm{UFC} / \mathrm{g}$ & $<10^{2} \mathrm{UFC} / \mathrm{g}$ \\
\hline $\mathrm{E}$ & $<10^{2} \mathrm{UFC} / \mathrm{g}$ & $<10^{2} \mathrm{UFC} / \mathrm{g}$ & $<10^{2} \mathrm{UFC} / \mathrm{g}$ & $<10^{2} \mathrm{UFC} / \mathrm{g}$ \\
\hline $\mathrm{F}$ & $<10^{2} \mathrm{UFC} / \mathrm{g}$ & $<10^{2} \mathrm{UFC} / \mathrm{g}$ & $<10^{2} \mathrm{UFC} / \mathrm{g}$ & $<10^{2} \mathrm{UFC} / \mathrm{g}$ \\
\hline G & $<10^{2} \mathrm{UFC} / \mathrm{g}$ & $<10^{2}$ UFC/g & $<10^{2}$ UFC/g & $<10^{2} \mathrm{UFC} / \mathrm{g}$ \\
\hline
\end{tabular}

*UFC/g: unidade formadora de colônia por grama

A amostra B apresentou contagens para Coliformes a $45{ }^{\circ} \mathrm{C}$ de $<10 \mathrm{UFC} / g$ e as demais amostras apresentaram resultados $<10^{2} \mathrm{UFC} / \mathrm{g}$ estando dentro dos padrões microbiológicos e 
sanitários para alimentos regulamentados pela Resolução RDC n 12 (ANVISA, 2001), cujos limites máximos para coliformes a $45^{\circ} \mathrm{C}$ são de $5 \times 10^{3} \mathrm{UFC} / \mathrm{g}$ ou $\mathrm{mL}$.

QUINTANA \& CARNEIRO (2007) avaliando a condições higiênico-sanitárias do queijo tipo Mussarela produzido na cidade de Morrinhos - GO, encontraram o valor máximo de 1,7 x $10^{1}$ UFC/g para os coliformes nas amostras analisadas, sendo abaixo do valor máximo permitido. PIETROWSKI et al. (2008) monitoraram a qualidade microbiológica de queijo tipo Mussarela comercializado na cidade de Ponta Grossa - PR e os resultados das análises para Coliformes a $45^{\circ} \mathrm{C}$ revelaram que 100\% das amostras encontram-se dentro do padrão. Esses estudos apresentam resultados que vem de encontro aos relatados no presente trabalho.

Para os resultados obtidos da Contagem de Staphylococcus coagulase positiva, a marca B apresentou resultado < $10 \mathrm{UFC/g}$ e às demais marcas apresentaram os resultados $<10^{2} \mathrm{UFC} / \mathrm{g}$, estando dentro dos padrões microbiológicos e sanitários para alimentos regulamentados pela Resolução RDC no 12 (ANVISA, 2001), cujos limites máximos são $10^{3}$ UFC/g.

QUINTANA \& CARNEIRO (2007) avaliando a contagem de Staphylococcus coagulase positiva, concluíram que nenhuma das amostras analisadas apresentou resultados acima da legislação, da mesma maneira que ocorreu no presente estudo. Já Pietrowski et al. (2008) em seu estudo obteve 3 amostras com valores acima de $10^{3}$ UFC/g. MONTEIRO \& BADARÓ (2006) avaliaram a qualidade microbiológica de queijos tipo Minas Frescal comercializados na cidade de Ipatinga - MG e para as análises de Staphylococcus, 92,8\% dos resultados foram positivos para o teste de coagulase.

A contaminação da maioria das amostras de queijos coloniais por Staphylococcus coagulase positiva pode ser explicada pelo fato de as principais fontes de contaminação do queijo ser a matériaprima e a manipulação por pessoas portadoras desse microrganismo (BRANT et al., 2007). A Tabela 2 expressa os resultados da análise de Salmonella sp. para o queijo tipo Mussarela.

Tabela 2 - Análises de Pesquisa de Salmonella sp. em queijo tipo Mussarela comercializado na região oeste do Paraná

\begin{tabular}{ccc}
\hline Amostra & \multicolumn{2}{c}{ Salmonella sp. } \\
& $1^{\text {o repetição }}$ & $2^{\circ}$ repetição \\
\hline A & Ausência & Ausência \\
B & Ausência & Ausência \\
C & Ausência & Ausência \\
D & Ausência & Ausência \\
E & Ausência & Ausência \\
F & Ausência & Ausência \\
G & Ausência & Ausência \\
\hline
\end{tabular}


De acordo com a RDC n 12 (ANVISA, 2001) a Salmonella sp. deve estar ausente em 25 g de amostra, e desta maneira todas as sete marcas analisadas encontram-se de acordo com a legislação vigente.

PIETROWSKI et al. (2008) em seu estudo observou que uma das amostras analisadas estava fora do padrão. Já Ribeiro (2003) analisando a qualidade microbiológica do queijo tipo Mussarela fatiado disponível em supermercados da cidade de Ponta Grossa - PR obteve 100\% de suas amostras dentro do padrão vigente e OLIVIERI (2004) que estudou a qualidade microbiológica de amostras de mercado de queijo tipo Mussarela, elaborado a partir de leite de búfala, não detectou a presença do mesmo microrganismo em nenhuma das amostras observadas.

A ausência de Salmonella sp. nas amostras indica um processamento adequado. Além disso, essa bactéria não é uma boa competidora, sofrendo injúria em meios ácidos ou com a presença de coliformes, principalmente, se a contaminação inicial for com um número pequeno de células. Nestas condições, esses microrganismos podem desaparecer ou permanecer em números indetectáveis em alimentos ácidos ou muito contaminados (BRASIL, 2003).

Os resultados da contagem de Coliformes a $45^{\circ} \mathrm{C}$ e contagem de Staphylococcus coagulase positiva para o queijo Colonial estão dispostos na Tabela 3.

Tabela 3 - Análise Microbiológica de Coliformes a $45^{\circ} \mathrm{C}$ e Staphylococcus coagulase positiva, de queijos Colonial comercializados na região oeste do Paraná

\begin{tabular}{|c|c|c|c|c|}
\hline \multirow[t]{2}{*}{ Amostra } & \multicolumn{2}{|c|}{$\begin{array}{c}\text { Análise de Coliformes } \\
\text { a } 45^{\circ} \mathrm{C} \\
\end{array}$} & \multicolumn{2}{|c|}{$\begin{array}{c}\text { Análise de Staphylococcus } \\
\text { coagulase positiva }\end{array}$} \\
\hline & $1^{\circ}$ repetição & $2^{\circ}$ repetição & $1^{\circ}$ repetição & $2^{\circ}$ repetição \\
\hline $\mathrm{H}$ & $<10^{2} \mathrm{UFC} / \mathrm{g}$ & $<10^{2} \mathrm{UFC} / \mathrm{g}$ & $<10^{2} \mathrm{UFC} / \mathrm{g}$ & $<10^{2}$ UFC/g \\
\hline I & $<10^{2} \mathrm{UFC} / \mathrm{g}$ & $<10^{2} \mathrm{UFC} / \mathrm{g}$ & $4,3 \times 10^{4} \mathrm{UFC} / \mathrm{g}$ & $2,5 \times 10^{4} \mathrm{UFC} / \mathrm{g}$ \\
\hline $\mathrm{J}$ & $<10^{2} \mathrm{UFC} / \mathrm{g}$ & $<10^{2} \mathrm{UFC} / \mathrm{g}$ & $3,0 \times 10^{2} \mathrm{UFC} / \mathrm{g}$ & $3,0 \times 10^{2} \mathrm{UFC} / \mathrm{g}$ \\
\hline $\mathrm{K}$ & $8,8 \times 10^{7} \mathrm{UFC} / \mathrm{g}$ & $<10^{3} \mathrm{UFC} / \mathrm{g}$ & $<10^{2} \mathrm{UFC} / \mathrm{g}$ & $<10^{2} \mathrm{UFC} / \mathrm{g}$ \\
\hline $\mathrm{L}$ & $<10^{3} \mathrm{UFC} / \mathrm{g}$ & $2,0 \times 10^{6} \mathrm{UFC} / \mathrm{g}$ & $<10^{2} \mathrm{UFC} / \mathrm{g}$ & $<10^{2} \mathrm{UFC} / \mathrm{g}$ \\
\hline M & $<10^{3} \mathrm{UFC} / \mathrm{g}$ & $4,1 \times 10^{9} \mathrm{UFC} / \mathrm{g}$ & $<10^{2} \mathrm{UFC} / \mathrm{g}$ & $<10^{2} \mathrm{UFC} / \mathrm{g}$ \\
\hline $\mathrm{N}$ & $<10^{3} \mathrm{UFC} / \mathrm{g}$ & $9,0 \times 10^{8} \mathrm{UFC} / \mathrm{g}$ & $5,4 \times 10^{4} \mathrm{UFC} / \mathrm{g}$ & $<10^{2} \mathrm{UFC} / \mathrm{g}$ \\
\hline
\end{tabular}

*UFC/g: unidade formadora de colônia por grama

Segundo a RDC $\mathrm{n}^{\circ} 12$ (ANVISA, 2001) o limite máximo permitido para coliformes termotolerantes em queijo Colonial é de 5,0 x $10^{3} \mathrm{UFC} / \mathrm{g}$, onde o resultado na primeira repetição da amostra K apresentou 8,8 x $10^{7} \mathrm{UFC/g}$, acima do limite estabelecido pela legislação, sendo que na segunda repetição apresentou resultado $<10^{3} \mathrm{UFC/g}$. Já as amostras L, M e N na segunda repetição apresentaram resultados acima dos limites estabelecidos pela legislação, sendo de 2,0 x 106; 4,1 x $10^{9}$ e $9,0 \times 10^{8} \mathrm{UFC} / \mathrm{g}$, respectivamente. Para as demais amostras os resultados foram $<10^{3} \mathrm{UFC} / \mathrm{g}$. 
IDE \& BENEDET (2001) observaram a qualidade do queijo Colonial produzido na região serrana do estado de Santa Catarina, encontrando uma grande variação nos resultados. Das 20 amostras analisadas, 10 estavam contaminadas na faixa de $1,5 \times 10^{6}$ a 4,6 x $10^{7} \mathrm{UFC} / \mathrm{g}$ de amostra e 10 na faixa de $1,5 \times 10^{3}$ a 9,3 x $10^{5}$ UFC/g de amostra, sendo que estes valores encontram-se próximos do presente trabalho.

ZAFFARI et al. (2007) monitoraram a qualidade bacteriológica de queijos artesanais comercializados em estradas do litoral norte do Rio Grande do Sul, onde que dos 29 estabelecimentos, 27 comercializavam queijos que apresentavam contagens de coliformes fecais acima dos valores estabelecidos na legislação vigente. Da análise desses resultados, pode-se inferir que a maioria dos queijos comercializados nestes estabelecimentos não apresenta condições sanitárias para consumo.

Segundo a RDC no 12 (ANVISA, 2001) o limite máximo permitido para Staphylococcus coagulase positiva é de $10^{3} \mathrm{UFC/g}$ e os resultados obtidos na amostra I na primeira repetição foi de 4,3 $\times 10^{4} \mathrm{UFC} / \mathrm{g}$ e na segunda repetição foi de $2,5 \times 10^{4} \mathrm{UFC} / \mathrm{g}$ estando acima do limite estabelecido pela legislação. A amostra $\mathrm{N}$ na primeira repetição apresentou resultados de 5,4 x $10^{4} \mathrm{UFC} / \mathrm{g}$ apresentando-se acima do limite. As demais amostras apresentaram resultados $<10^{2} \mathrm{UFC} / \mathrm{g}$ estando dentro dos limites estabelecidos pela legislação.

LUCAS et al. (2008) realizaram a caracterização microbiológica de queijo Colonial da região Oeste do Paraná e para análise de Staphylococcus coagulase positiva observaram que apenas as amostras do Laticínio $\mathrm{C}$ encontravam-se todas dentro dos limites estabelecidos, enquanto que para os outros laticínios, encontraram-se percentagens de 80 e 60 \% das amostras dos Laticínios A e B em desacordo com a legislação vigente, respectivamente. Na Tabela 4 encontram-se os resultados da análise de Salmonella sp. para o queijo Colonial.

Tabela 4 - Análises de Pesquisa de Salmonella sp. em queijos Colonial comercializados na região oeste do Paraná

\begin{tabular}{ccc}
\hline Amostra & & Salmonella sp. \\
& $1^{\text {o }}$ repetição & $2^{\text {o }}$ repetição \\
\hline H & Ausência & Ausência \\
I & Ausência & Ausência \\
J & Ausência & Ausência \\
K & Ausência & Ausência \\
L & Ausência & Ausência \\
M & Ausência & Ausência \\
N & Ausência & Ausência \\
\hline
\end{tabular}


Para a Pesquisa Salmonella sp. segundo a RDC nº 12 (ANVISA, 2001) é necessário que esta esteja ausente em 25 g, onde o resultado para todas as amostras e repetições apresentaram-se dentro das limites estabelecidos pela legislação. Este estudo difere de HOFFMANN (2003) que observou a qualidade microbiológica de queijos tipo "Minas Frescal”, vendidos em feiras livres na região de São José do Rio Preto - SP, onde 80\% das amostras apresentavam a presença de Salmonella sp. Já KOTWITZ \& GUIMARÃES (2003) monitoraram a qualidade microbiológica de queijos Coloniais produzidos no estado de Paraná e obtiveram 100\% de suas análises dentro dos padrões exigidos pela legislação, igualmente aos resultados encontrados neste estudo.

\title{
4. Conclusão
}

Tendo em vista os resultados obtidos neste trabalho, todas as amostras de queijo Mussarela encontram-se aptas para o consumo. No entanto a qualidade do queijo Colonial comercializado na região oeste do Paraná encontra-se inadequada, pois algumas amostras apresentam valores acima da legislação vigente.

\begin{abstract}
I have in mind the great consumption of the cheeses type Mussarela and Colonial cheese is made necessary to the evaluation of the microbiological quality of this. The study was aimed in valued the microbiological quality of seven samples of cheese type Mussarela and seven samples of Colonial cheese marketed in the western region of the Paraná. Being collected in two different periods totalizing 28 samples, when the next microbiological analyses are happening: Counting of Coliformes to $45^{\circ} \mathrm{C}$, Counting of Staphylococcus coagulase makes positive and Salmonella sp. where the analysed samples of the cheese were type Mussarela inside the limits established by the legislation in force, for the Colonial cheese one thinks the samples out of the limit.
\end{abstract}

Key-words: coliforms, current legislation, cheese.

\section{Referências}

ANVISA. Ministério da Saúde, Agência Nacional de Vigilância Sanitária, Resolução nº 12, de 02 de Janeiro de 2001. Aprova o regulamento técnico sobre padrões microbiológicos para alimentos. Diário Oficial da União, Brasília, DF, 02 jan. 2001. $51 \mathrm{p}$.

BRANT, L. M. F.; FONSECA, L. M.; SILVA, M. C. C. Avaliação da qualidade microbiológica do queijo-de-minas artesanal do Serro-MG. Arq. Bras. Med. Vet. Zootec., vol.59, n.6, p.1570-1574. 2007.

BRASIL. Instrução Normativa nº62, de 26 de agosto de 2003, do Ministério da Agricultura, do Abastecimento e da Reforma Agrária. Métodos analíticos oficiais para análises microbiológicas para controle de produtos de origem animal e água. Diário Oficial da União, Brasília, DF, 26 ago. 2003.

CARVAlHO, F. A. de; ALBUQUERQUE, L. C. de. Marketing e Qualidade Total - Fatores Competitivos para a indústria Laticinista. Juiz de Fora: Concorde. 1996. 
DREYFUSSO, J. L; RIBEIRO, L; DE MELO, R.; FIORESE, M. L; REITER, M. G. R. Qualidade microbiológica do queijo Minas Colonial comercializados em Blumenau-SC, In: Encontro Regional Sul de Ciências e Tecnologia de Alimentos, 7, Curitiba-PR-BR. Anais... Curitiba: 1 CD-ROM. 2001.

FURTADO, M. M. Principais problemas dos queijos causas e prevenção. São Paulo: Fonte Comunicações, 1999.

HOBBS, B. C; ROBERTS, D. Toxinfecções e controle Higiênico-Sanitário de Alimentos. 1. ed. São Paulo: Livraria Varela. 1998. 64p.

HOFFMANN, F. L.; SILVA, J. V. Qualidade Microbiológica de queijos tipo “Minas Frescal”, vendidos em feiras livres na região de São José do Rio Preto - SP. Revista Higiene Alimentar, São Paulo, v.6, n.96, p.89-94. 2003.

IDE, L. P. A.; BENEDET, H. D. Contribuição ao conhecimento do queijo colonial produzido na região serrana do estado de Santa Catarina, Brasil. Revista Ciência e Agrotecnologia, Lavras, v.25, p.1351-1358. 2001.

KOTTWITZ, L. B. M.; GUIMARÃES, I. M. Avaliação Microbiológica de queijos coloniais produzidos no estado de Paraná. Revista Higiene Alimentar, São Paulo, v.17, n.114/115, p.77-80. 2003.

LUCAS, S. D. M; TSUCHIYA, A. C.; SOUZA, M.; MATTANA, A.; PEREIRA, C. Caracterização microbiológica de queijo colonial da região oeste do Paraná, In: V Encontro Nacional de Difusão Tecnológica, 5, Medianeira-PR-BR. Anais... Medianeira: 1 CD-ROM. 2008.

MONTEIRO, M. V.; BADARÓ, A. C. L. Qualidade Microbiológica de Queijos tipo Minas Frescal comercializados na cidade de Ipatinga, Minas Gerais. Revista Higiene Alimentar, São Paulo, v.21, n.150, p.230-231. 2006.

OLIVIERI, D. A. Avaliação da Qualidade Microbiológica de amostras de mercado de Queijo Mussarela, elaborado a partir de leite de búfala. São Paulo. 2004.

PIETROWSKI, G. A. M.; RANTHUM, M.; CROZETA, T.; JONGE, V. Avaliação da qualidade microbiológica de queijo tipo Mussarela comercializado na cidade de Ponta Grossa, Paraná. Revista Brasileira de Tecnologia Agroindustrial, Ponta Grossa, v.2, n.2, p.25-31. 2008.

QUINTANA, R. C.; CARNEIRO, L. C. Avaliação das condições higiênico-sanitárias dos queijos minas frescal e mussarela produzidos na cidade de Morrinhos - GO. Revista Brasileira de Saúde e Produção Animal, v.8, n.3, p.205211. 2007.

RIBEIRO, C. C. Análise da Qualidade Microbiológica do Queijo Mussarela Fatiado Disponível em Supermercados da Cidade de Ponta Grossa, no Estado do Paraná. Ponta Grossa. 2003.

VALLE, J. L. E.; CAMPOS, S. D. S.; YOTSUNAGI, K; SOUZA, G. Influência do teor de gordura nas propriedades funcionais do queijo tipo mozarela. Revista Ciência e Tecnologia de Alimentos, Campinas, v.24, n.4, p.669-673, out./dez. 2004.

ZAFFARI, C. B.; MELLO, J. F.; DA COSTA, M. Qualidade bacteriológica de queijos artesanais comercializados em estradas do litoral norte do Rio Grande do Sul, Brasil. Revista Ciência Rural, Santa Maria, v.37, n.3, p.862-867, mai.jun. 2007.

Nome completo: Eliane Hermes

Filiação institucional: Universidade Estadual do Oeste do Paraná

Departamento: Centro de Ciências Exatas e Tecnológicas

Função ou cargo ocupado: Bolsista de Mestrado

Titulação: Tecnóloga Ambiental

Endereço: Rua do Aleijadinho 204, Jardim Universitário, Cascavel, Paraná, Brasil. CEP: 85819380

Telefones para contato: (45) 3035-7241 
e-mail: elianehermes@yahoo.com.br

Nome completo: Francielly Torres dos Santos Koelln

Filiação institucional: Universidade Tecnológica Federal do Paraná

Departamento: Tecnologia em Alimentos

Função ou cargo ocupado: Monitora de química

Titulação: Tecnóloga em Industrialização de Carnes

Endereço:

Avenida Brasil 4232, Bairro Parque Independência, Medianeira, Paraná, Brasil. CEP 85884-000

Telefones para contato: (45) 8808-7370

e-mail: franciellykoelln@yaho.com.br

Nome completo: Ademir Mattana

Filiação institucional: Universidade Tecnológica Federal do Paraná

Departamento: Gerec

Função ou cargo ocupado: Técnico do Laboratório de Análises Microbiologicas e físico-química de Alimentos e água - LAMAG - UTFPR

Titulação: Especialista em desenvolvimento de novos produtos alimentícios. Tecnólogo em Alimentos.

Endereço: Avenida Brasil 4232, Bairro Parque Independência, Medianeira, Paraná, Brasil. CEP 85884-000

Telefones para contato: $3240-8054$

e-mail: mattana@utfpr.edu.br 